\section{Unilateral Morbihan syndrome}

\author{
Laura Macca, ${ }^{1}$ Federica Li Pomi, ${ }^{1}$ \\ Alfonso Motolese, ${ }^{1}$ Paolo Broganelli, ${ }^{2}$ \\ Francesco Borgia ${ }^{1}$
}

${ }^{1}$ Department of Clinical and

Experimental Medicine, Section of

Dermatology, University of Messina;

${ }^{2}$ Dermatology Unit, City of Health and

Science of Turin, Italy

\begin{abstract}
Morbihan Syndrome is an uncommon condition characterized by the slow appearance of erythema and solid edema on the upper portion of the face. The disease is considered an end-stage complication of rosacea's recurrent episodes of vascular dilation and inflammation or a complication of acne vulgaris. The disease is often insidious to diagnose and challenging to treat We report a paradigmatic case of unilateral Morbihan Syndrome induced by use of fullface CPAP masks for OSAS in a patient with rosacea. OSAS could play a role in the development of rosacea symptoms. Our aim is to remark the importance of a careful, prolonged follow-up to optimize patient's management and to improve the adherence to therapy.
\end{abstract}

\section{Introduction}

Morbihan syndrome (MS), also known as Rosaceous lymphedema or Solid persistent facial edema, is a rare entity characterized clinically by chronic erythematous edema of the upper portion of the face (forehead, glabella, eyelids, and cheeks). ${ }^{1}$ It is considered a clinical variety or a complication of acne or rosacea. The cutaneous lesions persist indefinitely with no tendency to spontaneous involution finally resulting in deformity of facial contours and even in visual impairments. ${ }^{2}$ Caucasian adults of both sexes are affected. ${ }^{1}$ The diagnosis is clinical, and the histology is often non-specific. Etiopathogenesis is not completely known as well as its treatment management. Oral isotretinoin is considered first line therapy, although only few patients improve. We report a case of a severe and refractory MS in a 45-year-old Caucasian man.

\section{Case Report}

A 45-year-old obese male presented with a two-year history of asymptomatic tense elastic swelling of the right eyelid, gradually spreading to the cheek and forehead. Clinical examination revealed erythemato-telangiectatic rosacea of the nose, forehead and cheeks. Right eyelid was deformed by prominent non-depressive solid edema, partially involving the glabella and the dorsa of the nose, with alteration of facial contours and visual impairment. The left eyelid was normal (Figure 1). Because of Obstructive Sleep Apnea Syndrome (OSAS) since 2017 he started wearing every night a full-face Continuous Positive Airway Pressure (CPAP) ventilation mask. Patient referred that he used to sleep on the right side and those facial symptoms developed after starting CPAP.

Laboratory tests were negative or within normal ranges. A computed tomography revealed severe edema, but no other abnormalities.

Based on the anamnesis and on the typical clinical picture, the diagnosis of unilateral CPAP-induced Morbihan Syndrome (MS) was established. Differential diagnoses including periorbital metastases or congenital, infectious, inflammatory, neoplastic and miscellaneous diseases, were excluded. ${ }^{1}$

Treatment with oral isotretinoin 0.3 $\mathrm{mg} / \mathrm{kg} /$ day was prescribed. An appropriate dietary regimen was recommended to reduce weight and consequently OSAS symptoms and CPAP use. The patient was also invited to use intranasal devices instead of CPAP mask. At 6 months' follow-up, edema and infiltration were only slightly decreased. He was not compliant with dietary regimen (Figure 2). The intranasal devices resulted ineffective to control OSAS symptoms. Oral treatment was stopped because of lack of significant improvement and onset of side effects such as skin dryness and chest pain. Surgical treatment was proposed to debulk the enlarged tissue of lymphoedema.

\section{Discussion}

MS is an uncommon condition characterized by the slow appearance of erythema and solid edema on the upper portion of the face. ${ }^{2}$ The disease is considered an endstage complication of rosacea's recurrent episodes of vascular dilation and inflammation or a complication of acne vulgaris. ${ }^{2}$ It is supposed that chronic inflammation may lead to the remodeling of vascular and lymphatic vessels through the destruction of
Correspondence: Laura Macca, Department of Clinical and Experimental Medicine, Section of Dermatology, University of Messina, Italy. Tel.: +39.0902213933

E-mail: lauramacca7@gmail.com

Key words: Morbihan disease, Solid persistent facial edema, Continuous positive airway pressure, Obstructive sleep apnea, Rosacea.

Contributions: LM and FLP: first authors, wrote the manuscript. AM: collected the data of the patient. PB: was responsible for the computed tomography. FB: served as a principal investigator, established the diagnosis, and was responsible for revision.

Conflict of interest: The authors declare no potential conflict of interest.

Funding: None.

Ethical statement and informed consent: The patient gave consent to use his personal data for the publication of this case report and any accompanying images.

Please cite this article as: Macca L, Li Pomi $F$, Motolese A, et al. Unilateral Morbihan syndrome. Dermatol Rep 2022;14:9270.

Received for publication: 20 May 2021.

Accepted for publication: 7 July 2021.

This work is licensed under a Creative Commons Attribution-NonCommercial 4.0 International License (CC BY-NC 4.0).

(C) Copyright: the Author(s), 2021

Licensee PAGEPress, Italy

Dermatology Reports 2022; 14:9270

doi:10.4081/dr.2021.9270

Publisher's note: All claims expressed in this article are solely those of the authors and do not necessarily represent those of their affiliated organizations, or those of the publisher, the editors and the reviewers. Any product that may be evaluated in this article or claim that may be made by its manufacturer is not guaranteed or endorsed by the publisher.

collagen and elastic fibers around the blood vessels, with consequent increase of vascular permeability and fluid loss. ${ }^{3}$ Chronic obstruction of lymphatic vessels or mast cell-induced fibrosis in the dermis could in turn cause lymphedema of the underlying tissue. ${ }^{3}$ The disease is often insidious to diagnose and challenging to treat. It can produce deformity that may lead to visual impairment, constant tearing from the affected eye, functional problems, and diminished self-esteem as a result of cosmetic disfigurement. ${ }^{4}$ Our patient was not able to completely open his eye and each time he came to visit he seemed concerned 
with no expectation about cosmetic improvement. It is worth to mention that solid persistent facial edema represents a treatment challenge. ${ }^{4}$ In order to reduce the emotional suffering of the patient, every therapeutic attempt should be considered. Several options have been proposed such as isotretinoin, systemic antibiotics (tetracyclines, metronidazole), antihistamines (ketotifen) and systemic corticosteroids (prednisone, prednisolone). ${ }^{4}$ Clofazimine and thalidomide are also reported. ${ }^{3}$ Overall, the outcome of the available treatments is unsatisfactory. Isotretinoin is considered the first-line therapy, although about $20 \%$ of patients experiences no improvement. ${ }^{1}$ It is usually associated to systemic corticosteroids, but in our case, it was contraindicated because of patient's comorbidities (hypertension, hyperinsulinemia, obesity,
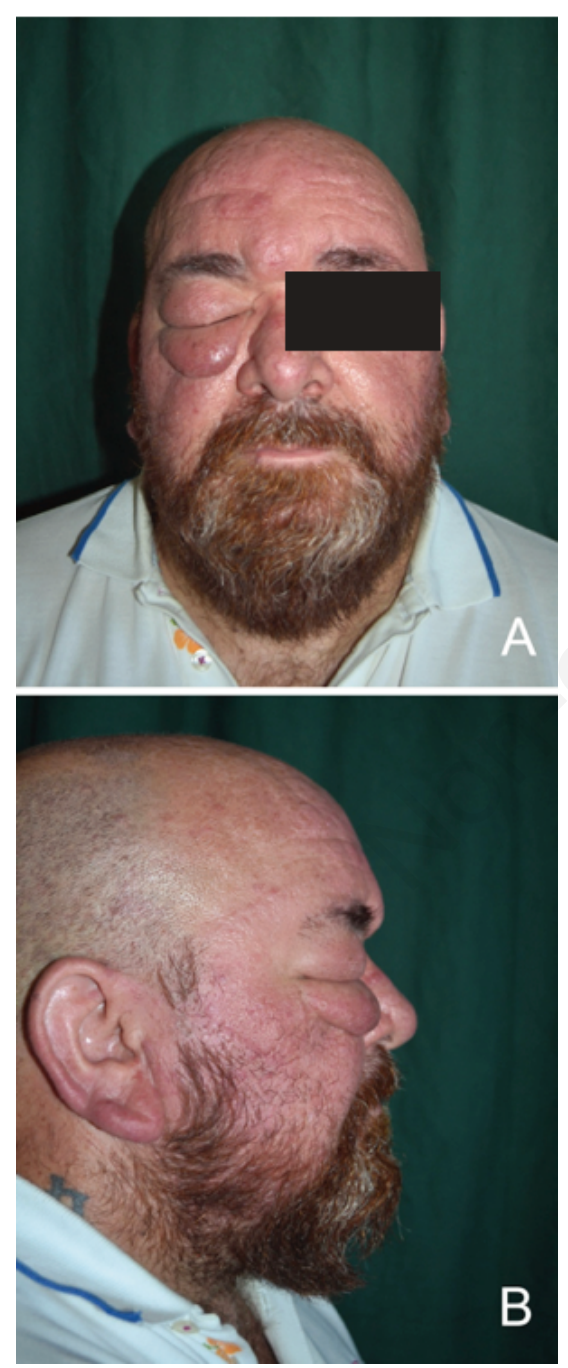

Figure 1. Frontal and side view of the face before starting treatment. Severe nondepressive solid edema of the right lid, partially involving the glabella and the dorsa of the nose. and metabolic syndrome). Surgery treatment could be suggested as a valuable alternative to chronic use of systemic therapy. As a complement to these medical and surgical treatments, it is essential to reduce predisposing factors such as obesity and CPAP use. Some comorbidities represent an important link between CPAP use for OSAS and rosacea. OSAS is associated with metabolic syndrome. Interestingly, rosacea is also associated with metabolic syndrome. ${ }^{5}$ So, OSAS could play a role in the development of rosacea symptoms. ${ }^{5}$ It is therefore essential to promote patient weight loss to reduce OSAS and rosacea symptoms. Because of patient's low compliance to the dietary regimen, OSAS worsened. Therefore, he continued to use CPAP developing this chronic progressively disabling disease.
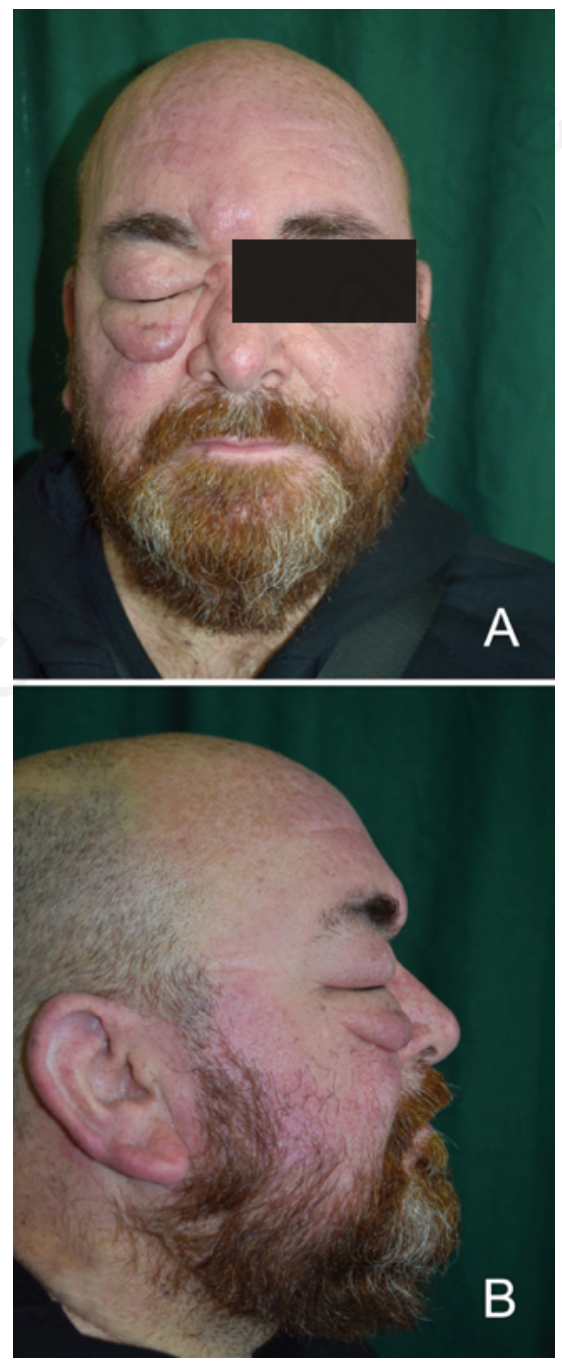

Figure 2. Frontal and side view of the face after six months of treatment with oral isotretinoin.

\section{Conclusions}

MS should be considered in patients with previous diagnosis of rosacea and concomitant CPAP use. Its early recognition and prompt treatment is mandatory to reduce the risk of progression and to avoid negative impact on patient's quality of life. However, an optimal therapeutic response is desirable only when it is associated with full compliance of the patient and the control of the predisposing causes.

\section{References}

1. Veraldi S, Persico MC, Francia C. Morbihan syndrome. Indian Dermatol Online J 2013;4:122-4

2. Yvon C, Mudhar HS, Fayers T, et al. Morbihan Syndrome, a UK Case Series. Ophthalmic Plast Reconstr Surg 2020;36:438-43.

3. Boparai RS, Levin AM, Lelli GJ Jr. Morbihan disease treatment: two case reports and a systematic literature review. Ophthalmic Plast Reconstr Surg 2019;35:126-32.

4. Heibel HD, Heibel MD, Cockerell CJ. Successful treatment of solid persistent facial edema with isotretinoin and compression therapy. JAAD Case Rep 2020;6:755-7.

5. Logger JGM, Peppelman M, van Vugt $\mathrm{R}$, Driessen RJB. Rosacea and use of continuous positive airway pressure mask for obstructive sleep apnea syndrome: report of five cases. Ann Dermatol 2020;32:247-50. 